Gut, 1989, 30, 541-545

Case report

\title{
Primary atypical mycobacteriosis of the intestine: a report of three cases
}

\author{
K FUJISAWA, $\mathrm{H}$ WATANABE, $K$ YAMAMOTO, T NASU, Y KitAhARA, \\ AND M NAKANO \\ From the Department of Internal Medicine, Department of Bacteriology, Kyushu Rosai Hospital, Kitakyushu, \\ Fukuoka, Japan, and Department of Pathology, School of Medicine, Niigata University, Niigata, Japan
}

Summary Primary atypical mycobacteriosis of the lung is comparatively common but primary atypical mycobacteriosis of the intestine had not been described previously in the English literature. We have treated three such patients with antituberculous drugs and the clinicopathological findings and course are described. We suggest that these findings may belong to a new clinical entity.

\section{Case reports}

These three Japanese patients did not have AIDS, had not been travelling abroad and had not had anal intercourse. Chest $x$-ray films, upper gastrointestinal and small bowel examinations showed no abnormalities except for a secondary dilatation of the terminal ileum of patient 1. Atypical mycobacteria were detected from the lesions of the large intestine and were varified in culture. Human and bovine tubercle bacilli were absent.

\section{PATIENT 1}

A 23 year old female typist was admitted complaining of dull pain in the right lower quadrant of the abdomen of several months duration. Erythema nodosum on the extremities and a hard granulomatous nodule in the perianus were present. Laboratory findings were: body temperature $38.1^{\circ} \mathrm{C}$, ESR $76 \mathrm{~mm} / \mathrm{h}$, CRP 5(+), anaemia (Hb $7.7 \mathrm{~g} / \mathrm{dl})$, leukocytosis (WBC 9300 cells $/ \mathrm{mm}^{3}$ ), platelet count was $50.6 \times 10^{4} / \mathrm{mm}^{3}$, fraction of $\alpha_{2}$-globulin $14.4 \%$. Other routine blood studies were normal. The Mantoux test was positive $(17 \times 25 \mathrm{~mm}$ in diameter $)$ in 48 hours. A barium enema examination showed a broad circular stenosis with a cobblestone appearance from the caecum to the ascending colon. The cobblestone appearance was arranged in the longitudinal direction in the stenotic lumen, barium flecks of 'rose-thorn ulcers' were seen in the outer margin of

Address for correspondence: Dr Kazuaki Fujisawa, Kyushu Rosai Hospital. 1-7-11-201, Kuzuhara-Takamatu, Kokura-Minami-ku. Kitakyushu, Fukuoka $8(0)-(1)$. Japan.

Accepted for publication 20) September 1988. the stenosis. A barium enema also revealed destruction of the Bauhin's valve, dilatation of the terminal ileum, and two skip lesions; ulcer scars in the right transverse colon and inflammatory polyps in the left transverse colon (Fig. 1a).

Colonoscopy revealed smooth spherical mucosal protrusions at the most distal part of the stenosis of the ascending colon, small in some places and large in others and the locations were irregular with multiple small ulcers. Colonoscopy also revealed cobblestone appearance in the hepatic flexure and left transverse colon, and ulcer scars in the right transverse colon. The colonoscope could not be passed through the stenotic lumen. Biopsy specimens taken from the ascending colon showed marked oedema and chronic inflammatory infiltrates including macrophages and monocytes, and non-caseous epithelioid cell granulomas with Langhans' giant cells (Fig. 2). A large number of acid fast bacteria were present in the biopsy material and in the local gut fluid stained with Ziehl-Neelsen stain, and the bacteria were confirmed by culture showing moderately long rods, straight or slightly curved, not showing banded or beaded forms, negative for niacin test: atypical mycobacterium. Mycobacterium tuberculosis was neither present in the smear and the gut fluid nor cultured. Skin biopsy from the extremities disclosed erythema nodosum. On the seventh hospital day the patient became afebrile and the erythema nodosum spontaneously disappeared.

Atypical mycobacterium was considered to be pathogenic and treatment with isoniazid, rifampicin, and streptomycin was started on the 13th hospital day and her general condition improved (Table). Twenty 


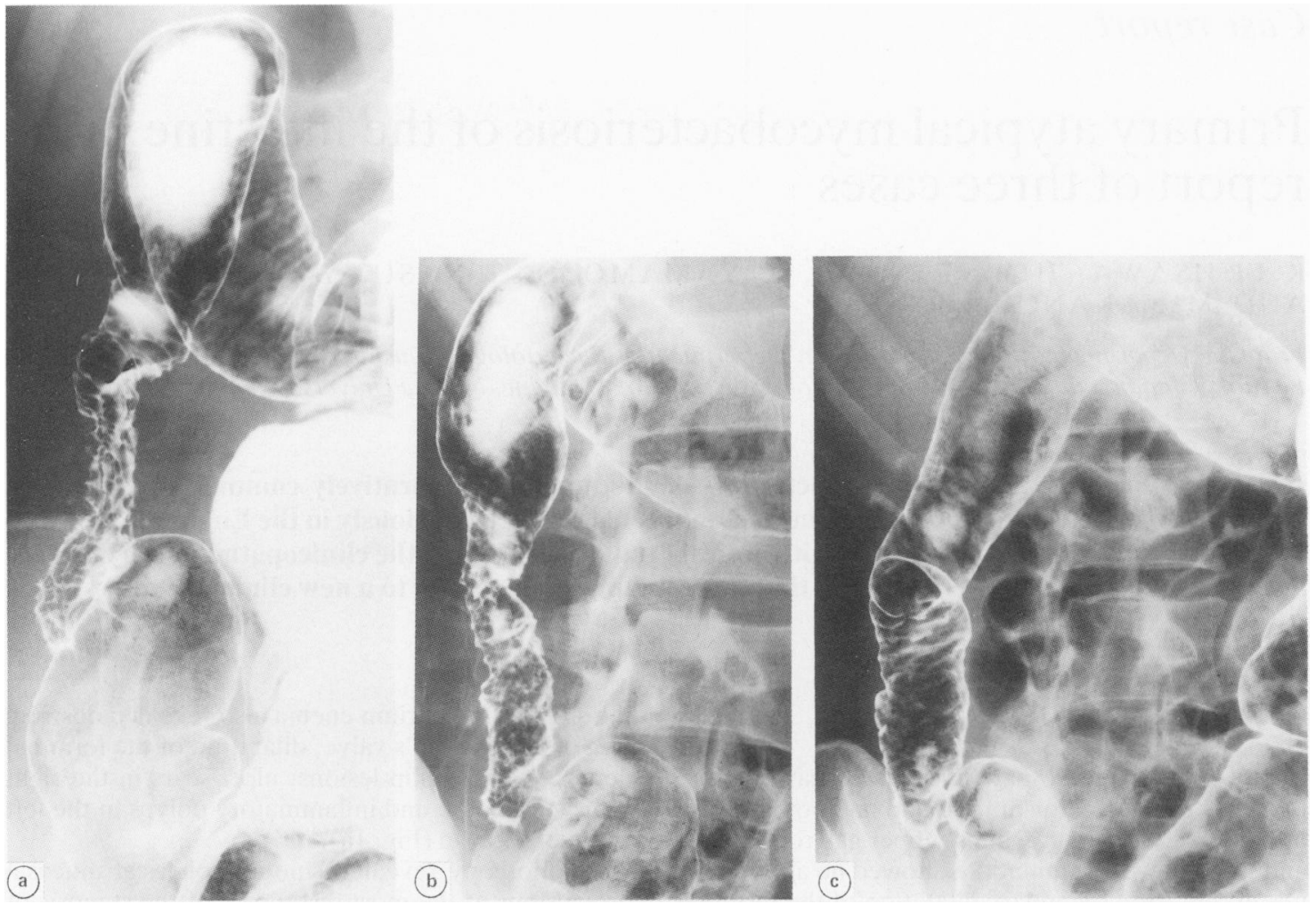

Fig. 1 Barium enema showed a broad circular stenosis with a cobblestone appearance from the caecum to the ascending colon, with destruction of the Bauhin's valve, with marked dilatation of the terminal ileum, and with ulcer scars in the righttransverse colon before treatment (a), and showed improvement of the stenosis and mucosal abnormalities of the colon with anti-tuberculous drugs treatment at four weeks (b) and at eight weeks (c). (Patient I.)

five days after start of this treatment, biopsy specimens were taken from the terminal ileum epithelioid cell granulomas were found to be present. The stenosis of the ascending colon was released, and biopsy specimens taken revealed moderate inflammatory infiltrates of plasma cells, lymphocytes, and eosinophilic cells, and a distinct decrease in number of macrophages and monocytes, and no epithelioid cell granuloma. A large number of the atypical mycobacterium were present in smears of the biopsy material of the ascending colon, however, and were verified by culture. Barium enema showed gradual improvement of the stenosis and mucosal abnormalities after treatment (Fig. 1b, c). Intramuscular injection of streptomycin $1 \mathrm{~g} /$ day has continued for six weeks, then twice a week for 14 weeks. Ninety one days after start of the treatment, a large number of the same atypical mycobacteria were present in smears of the biopsy material, ethambutol was added to this treatment. The granulomatous nodule in the perianus was surgically resected on 116th hospital day, and revealed infiltration of chronic inflamma- tory cells including monocytes and macrophages and a few epithelioid cell granulomas in the dermis, but no atypical mycobacterium was found in the tissue and the culture medium. One year after the initial therapy, a barium enema showed remarkable improvement in comparison with that of the eight weeks after treatment, and colonoscopy showed many ulcer scars instead of cobblestone appearance. No more atypical mycobacterium were present in

\section{Table Clinical course of the case I patient}

\begin{tabular}{lcccc}
\hline & & \multicolumn{3}{c}{ After treatment (weeks) } \\
\cline { 3 - 5 } & $\begin{array}{c}\text { Before } \\
\text { treatment }\end{array}$ & 2 & 4 & 8 \\
\hline ESR $(\mathrm{mm} \mathrm{1} \mathrm{h/2} \mathrm{h)}$ & $76 / 126$ & $50 / 97$ & $10 / 29$ & $3 / 8$ \\
CRP & $5(+)$ & $(+)$ & $(-)$ & $(-)$ \\
WBC (cells $\left./ \mathrm{mm}^{3}\right)$ & 9300 & 4100 & 4200 & 3500 \\
$\mathrm{Hgb}(\mathrm{g} / \mathrm{dl})$ & $7 \cdot 7$ & $9 \cdot 0$ & 12.9 & $13 \cdot 4$ \\
Plat $\left(\times 10^{4} / \mathrm{mm}^{3}\right)$ & $50 \cdot 6$ & $39 \cdot 0$ & 23.6 & 19.9 \\
\hline
\end{tabular}

Laboratory findings improved after treatment with isoniazid $4(0) \mathrm{mg}$. rifampicin $450 \mathrm{mg}$, and streptomycin $1 \mathrm{~g} /$ day. (Patient 1 .) 


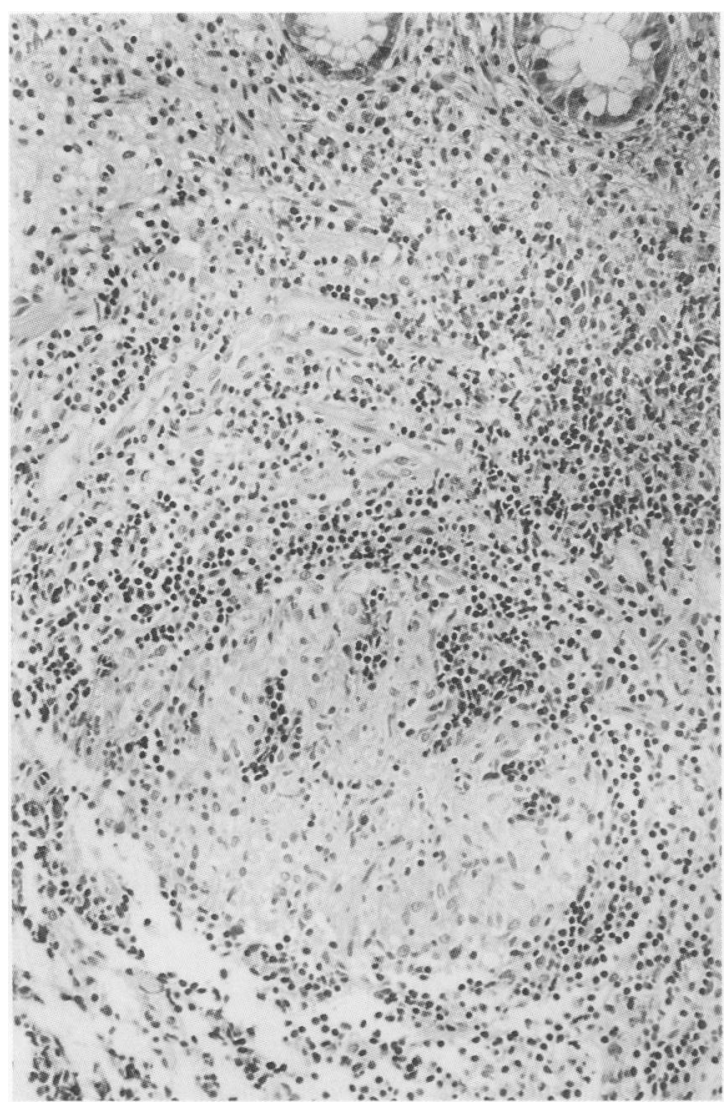

Fig. 2 Biopsy of the ascending colon showed a non-caseous epithelioid cell granuloma and chronic inflammatory infiltrates. (Patient 1.)

smears of the biopsy material nor cultured. The exact typing of atypical mycobacterium revealed Mycobacterium non-chromogenicum.

PATIENT 2

A 21 year old woman, a seamstress, was admitted to our hospital complaining of occasional fresh bleeding after defecation of several months duration. She underwent appendectomy at 15 years old, but had taken neither oral contraceptives not other drugs. Physical examination revealed no abnormalities and laboratory findings were normal. The Mantoux test was strongly positive $(18 \times 20 / 60 \times 100 \mathrm{~mm})$. A barium enema examination revealed niches in the rectum.

Colonoscopy disclosed four discrete oval and circular ulcers with necrotic white coat in the anterior wall of the lower rectum. The remaining rectal mucosa and the colonic mucosa were intact. Biopsy showed chronic inflammatory infiltrates, noncaseous epithelioid cell granuloma, and a large number of acid fast bacteria were present in ZiehlNeelsen stained smears of the biopsy material confirming atypical mycobacterium by culture. The species of atypical mycobacterium was also Mycobacterium nonchromogenicum. The rectal ulcers completely cured within two months treatment with isoniazid, rifampicin and ethambutol.

PATIENT 3

A 65 year old man was admitted complaining of general malaise and weight loss. He had had Rumsay-Hunt syndrome with left facial palsy and left hearing loss for four months. Laboratory findings were within the normal except for faecal occult blood. The Mantoux test was strongly positive $(13 \times 18 / 15 \times 20 \mathrm{~mm})$. A barium enema examination revealed a narrowing of the lumen with mucosal polypoid lesions, niches and diverticula in the midtransverse colon. There were many huge pseudodiverticula in the hepatic flexure, but no abnormal findings in other portions of the colon or in the rectum (Fig. 3). Colonoscopy showed a narrowing of the lumen with polypoid protrusions of the mucosa, ulcers and diverticula in the mid-transverse colon and many huge pseudodiverticula in the hepatic flexure. Biopsy specimens taken from the midtransverse colon showed non-caseous epithelioid cell granulomas and chronic inflammatory cells as well as macrophages and monocytes. A large number of acid fast bacteria were present in Ziehl-Neelsen stained smears of the biopsy material, and these were confirmed as atypical mycobacterium by culture. The species was identified as Mycobacterium chelonae subsp abscessus. Treatment with isoniazid, rifampicin, and streptomycin was effective and his general condition gradually improved.

\section{Discussion}

It has become clear that many mycobacteria are capable of causing disease in man.' It is now known that there are 54 species of atypical mycobacterium.? Water is emerging as an important source of the contamination,' because our three patients had neither basal diseases nor pulmonary atypical mycobacteriosis, ingestion of the organisms with food, water, or other contaminated materials may be possible modes of bowel infection."

Several investigators have commented on the morphologic similaritics that make it difficult to distinguish lesions caused by atypical mycobacteria from lesions by $M$ tuberculosis, while others believe that there are recognisable differences. ${ }^{4}$ The histology of our three cases revealed the non-cascous epithelioid cell granulomas with Langhans' giant cells and the infiltration of many macrophages and mono- 
cytes. After treatment, the histology showed marked infiltration of plasma cells and eosinophilic cells, and marked decrease in number of monocytes and macrophages as well as no detection of epithelioid cell granulomas. These findings are compatible with those of pulmonary atypical mycobacteriosis. In patient 1 , the granulomatous nodule in the perianus may be a primary focus of atypical mycobacterium through a minor injury or may be a metastatic focus or a secondary change, like erythema nodosum, of the intestinal lesion.

Secondary intestinal atypical mycobacteriosis is seen in severely immunocompromised states, such as AIDS, in which extensive infection of $M$ aviumintracellulare complex are more common. At sigmoidoscopy, the mucosa of the rectum and sigmoid colon is said to be oedematous, erythematous, and friable, with multiple linear and oval erosions. Intestinal biopsy may reveal pathologic findings that mimic Whipple's disease; macrophages laden with diastase-resistant, PAS-positive material fill the lamina propria, but the inciting organism is not Whipple's bacillus but $M$ avium-intracellulare complex. ${ }^{3}$ Granuloma formation is rarely caused by the absence of cell mediated immunity in those individuals. 'These findings of secondary atypical mycobacteriosis of the intestine are different from those of our patients with primary atypical mycobacteriosis.

Crohn's disease should be differentiated from patient 1 . The ascending colon in this patient had a cobblestone appearance, but protrusion of the mucosa was remarkably spherical and no longitudinal ulcer was seen. Bauhin's valve was destroyed, and there were diverse pathological changes in the mucosa from the active stage to the healing stage in the transverse colon. A large number of atypical mycobacteria were present in Ziehl-Neelsen stained smears of the biopsy material and these were confirmed by culture. The patient's general condition improved after treatment with anti-tuberculous drugs and from these data, a diagnosis of atypical mycobacteriosis seems likely.

In discrete, oval or circular rectal ulcerations as in patient 2, some differential diagnosis must be considered, such as simple, non-specific ulcer, focal 
ischaemic colonic ulcers and so on. Histological examination showed non-caseous epithelioid cell granuloma, the infiltration of many inflammatory cells and a large number of atypical mycobacteria in direct smears and in culture as well as the response to chemotherapy are diagnostic of atypical mycobacteriosis.

In patient $3 x$-ray and endoscopic findings showed a similarity to colonic tuberculosis. Histologic findings in the colonic biopsy were not so conclusive for a diagnosis of atypical mycobacteriosis. Therefore the Ziehl-Neelsen staining of biopsy smears and cultures are important for differential diagnosis.

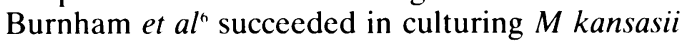
from a mesenteric lymph node of one patient with Crohn's disease. Chiodini et al' reported the isolation of an unclassified mycobacterium which is most closely related to $M$ paratuberculosis from resected gastrointestinal tissues obtained from two patients with Crohn's disease. Thayer et al ${ }^{\text {x }}$ described Crohn's disease patients with a statistically significant increase in antibody titre to $M$ paratuberculosis, as compared with healthy controls. They proposed that mycobacterium plays an aetiologic role in at least some cases of Crohn's disease. There are, however, many diverse opinions. ${ }^{9-11}$

As atypical mycobacterium may be present not only in patients with Crohn's disease but also in those with ulcerative colitis and even healthy persons, ${ }^{610}$ it is necessary to establish the criteria for diagnosing atypical mycobacteriosis. Yamamoto et al ${ }^{12}$ proposed diagnostic major criteria for disease caused by atypical mycobacteria, as follows: (1) repeated discharge (more than four times) of atypical mycobacteria in large numbers (more than 100 colonies) and presence of clinical symptoms that may be attributed to these bacilli; and (2) presence of a lesion containing atypical mycobacteria and the histopathologic changes possibly as a result of the bacilli. A diagnosis of the disease is made when either one of the two major criteria is satisfied. The findings in our three patients meet these major criteria. In addition, our patients' general condition improved after treatment with antituberculous drugs.

There is a possibility that some patients thought to have Crohn's disease or tuberculosis are indeed cases of atypical mycobacteriosis of the intestine. The clinician must be alert to this newly recognised presentation of primary atypical mycobacteriosis of the intestine which may belong to a new clinical entity.

We are grateful to Professor $\mathrm{H}$ Saito at Shimane Medical University for identification of atypical mycobacteria, and to Professor H Nawata, Kyushu University and to M Ohara for comments.

\section{References}

1 Wolinsky E. Nontuberculous mycobacteria and associated discase. Am Rev Respir Dis 1979; 119: 107-59.

2 Wayne LG, Kubica GP. The mycobacteria. In: Sneath PHA, Mair NS, Sharpe ME, Holt JG, cds. Bergey's manual of systematic bacteriology. Baltimore: Williams \& Wilkins, 1986: 1435-57.

3 Wolke A, Meyers S, Adelsberg BR, et al. Mycobacterium avium-intracellulare-associated colitis in a patient with the acquired immunodeficiency syndrome. J Clin Gastroenterol 1984; 6: 225-9.

4 Reid JD. Wolinsky E. Histopathology of lymphadenitis caused by atypical mycobacteria. Am Rev Respir Dis 1969; 99: 8-12.

5 Gillin JS, Urmacher C, West R, et al. Disseminated Mycobacterium avium-intracellulare infection in acquired immunodeficiency syndrome mimicking Whipple's disease. Gastroenterology 1983; 85: 1187-91.

6 Burnham WR. Lennard-Jones JE, Stanford JL, et al. Mycobacteria as a possible cause of inflammatory bowel disease. Lancet 1978; ii: 693-6.

7 Chiodini RJ, Kruiningen HJV, Thayer WR, et al. Possible role of mycobacteria in inflammatory bowel discase I. An unclassificd mycobacterium species isolated from patients with Crohn's disease. Dig Dis Sci 1984; 29: 1073-9.

8 Thayer WR Jr, Coutu JA, Chiodini RJ, et al. Possible role of Mycobacteria in inflammatory bowel disease II. Mycobacterial antibodies in Crohn`s disease. Dig Dis $S_{c i}$ 1984; 29: 1080-5.

9 Gitnick G. Is Crohn's disease a mycobacterial disease after all? Dig Dis Sci 1984; 29: 1086-8.

10 Graham DY, Markesich DC, Yoshimura HH. Mycobacteria and inflammatory bowel disease: result of culture. Gastroenterology 1987; 92: 436-42.

11 Whorwell PJ, Davidson IW, Beeken WL, et al. Search by immunofluorescence for antigens of rotavirus. pseudomonas maltophilia, and mycobacterium kansasii in Crohn's disease. Lancet 1978; ii: 697-8.

12 Yamamoto M, Ogura Y, Sudo K, et al. Diagnostic criteria for disease caused by atypical mycobacteria. $\mathrm{Am}$ Rev Respir Dis 1967; 96: 773-8. 